\title{
Using High-Performance Computing for the Modeling of High-Speed Dynamics
}

\author{
Arash Ramezani and Hendrik Rothe \\ Chair of Measurement and Information Technology, University of the Federal Armed Forces, Hamburg 22043, Germany
}

\begin{abstract}
Since computers and software have spread into all fields of industry, extensive efforts are currently made in order to improve the safety by applying certain numerical solutions. For many engineering problems involving shock and impact, there is no single ideal numerical method that can reproduce the various regimes of a problem. An approach wherein different techniques may be applied within a single numerical analysis can provide the "best" solution in terms of accuracy and efficiency. This paper presents a set of numerical simulations of ballistic tests which analyze the effects of soda lime glass laminates. The goal is to find an appropriate solver technique for simulating brittle materials and thereby improve bullet-proof glass to meet current challenges. To have the correct material model available is not enough. In this work, the main solver technologies are compared to create a perfect simulation model for soda lime glass laminates. The calculation should match ballistic trials and be used as the basis for further studies. These numerical simulations are performed with the nonlinear dynamic analysis computer code ANSYS AUTODYN.
\end{abstract}

Key words: Solver technologies, simulation models, brittle materials, optimization, armor systems.

\section{Introduction}

Main in the security sector, the partly insufficient safety of people and equipment due to failure of industrial components are ongoing problems that cause great concern. Since computers and software have spread into all fields of industry, extensive efforts are currently made in order to improve the safety by applying certain computer-based solutions. To deal with problems involving the release of a large amount of energy over a very short period of time, e.g., explosions and impacts, there are three approaches. As the problems are highly non-linear and require information regarding material behavior at ultra-high loading rates which is generally not available, most of the work is experimental and may cause tremendous expenses. Analytical approaches are possible if the geometries involved are relatively simple and if the loading can be described through boundary conditions, initial conditions, or a combination of the two.

Corresponding author: Arash Ramezani, Ph.D., research fields: numerical simulation and terminal ballistics. E-mail: ramezani@hsu-hh.de.
Numerical solutions are far more general in scope and remove any difficulties associated with geometry [1].

For structures under shock and impact loading, numerical simulations have proven to be extremely useful. They provide a rapid and less expensive way to evaluate new design ideas. Numerical simulations can supply quantitative and accurate details of stress, strain, and deformation fields that would be very costly or difficult to reproduce experimentally. In these numerical simulations, the partial differential equations governing the basic physics principles of conservation of mass, momentum, and energy are employed. The equations to be solved are time-dependent and nonlinear in nature. These equations, together with constitutive models describing material behavior and a set of initial and boundary conditions, define the complete system for shock and impact simulations.

The governing partial differential equations need to be solved in both time and space domains. The solution over the time domain can be achieved by an explicit method. In the explicit method, the solution at 
a given point in time is expressed as a function of the system variables and parameters, with no requirements for stiffness and mass matrices. Thus, the computing time at each time step is low but may require numerous time steps for a complete solution.

The solution for the space domain can be obtained utilizing different spatial discretizations, such as Lagrange [2], Euler [3], ALE (Arbitrary Lagrange Euler) [4], or mesh free methods [5]. Each of these techniques has its unique capabilities, but also limitations. Usually, there is not a single technique that can cope with all the regimes of a problem [6].

This work will focus on brittle materials and transparent armor (consisting of several layers of soda lime float glass bonded to a layer of polycarbonate to produce a glass laminate). Using a CAD (computer-aided design) neutral environment that supports direct, bidirectional and associative interfaces with CAD systems, the geometry can be optimized successively. Native CAD geometry can be used directly, without translation to IGES or other intermediate geometry formats [7].

The work will also provide a brief overview of ballistic tests to offer some basic knowledge of the subject, serving as a basis for the comparison and verification of the simulation results. Details of ballistic trials on transparent armor systems are presented. Here, even the crack formation must precisely match later simulations. It was possible to observe crack motion and to accurately measure crack velocities in glass laminates. The measured crack velocity is a complicated function of stress and of water vapor concentration in the environment [8].

The objective of this work is to compare current solver technologies to find the most suitable simulation model for brittle materials. Lagrange, Euler, ALE, and "mesh free" methods, as well as coupled combinations of these methods, are described and applied to a bullet-proof glass laminate structure impacted by a projectile.
The results shall be used to improve the safety of ballistic glasses. Instead of running expensive trials, numerical simulations should be applied to identify vulnerabilities of structures. Contrary to the experimental results, numerical methods allow easy and comprehensive studying of all mechanical parameters. Modeling will also help to understand how the transparent armor schemes behave during impact and how the failure processes can be controlled to our advantage. By progressively changing the composition of several layers and the material thickness, the transparent armor will be optimized.

After a brief introduction and description of the different methods of space discretization, there is a short section on ballistic trials, where the experimental set-up is depicted.

The last section describes the numerical simulations and the use of high-performance computing. These paragraphs of analysis are followed by a conclusion.

\section{State-of-the-Art}

First approaches for optimization were already developed in 1999. Mike Richards, Richard Clegg, and Sarah Howlett investigated the behavior of glass laminates in various configurations at a constant total thickness [9]. Resulting from the experimental studies, numerical simulations were created and adjusted to the experimental results using 2D-Lagrange elements only.

Pyttel, Liebertz and Cai explore the behavior of glass upon impact with three-dimensional Lagrange elements [10]. In 2011, these studies were used to analyze crash behavior.

In the same year, Zang and Wang dealt with the impact behavior on glass panels in the automotive sector [11]. In doing so, self-developed methods of numerical simulation were supposed to be compared with commercial codes. For the first time, mesh-free methods were applied, although these were not coupled with other solver technologies.

In this study, different methods for the simulation of safety glass will be introduced. In so doing, the 
possibility of coupling various solver technologies will be discussed and illustrated by means of an example. For the first time, glass laminates will be modeled using coupled methods. Techniques previously applied, show considerable shortcomings in portraying the crack and error propagation in the glass. Mesh-free approaches, in turn, do not correctly present the behavior of synthetic materials. To overcome the shortcomings of these single-method approaches, this paper will present an optimal solution to the problem by combining two methods.

\section{Methods of Space Discretization}

The spatial discretization is performed by representing the fields and structures of the problem using computational points in space, usually connected with each other through computational grids. Generally, the following applies: the finer the grid, the more accurate the solution. For problems of dynamic fluid-structure interaction and impact, there typically is no single best numerical method which is applicable to all parts of a problem. Techniques to couple types of numerical solvers in a single simulation can allow the use of the most appropriate solver for each domain of the problem [12]. The most commonly used spatial discretization methods are Lagrange, Euler, ALE (a mixture of Lagrange and Euler), and mesh-free methods, such as SPH (Smooth Particles Hydrodynamics) [13].

\subsection{Lagrange}

The Lagrange method of space discretization uses a mesh that moves and distorts with the material it models as a result of forces from neighboring elements (meshes are imbedded in material). There is no grid required for the external space, as the conservation of mass is automatically satisfied and material boundaries are clearly defined. This is the most efficient solution methodology with an accurate pressure history definition. The Lagrange method is most appropriate for representing solids, such as structures and projectiles. If however, there is too much deformation of any element, it results in a very slowly advancing solution and is usually terminated because the smallest dimension of an element results in a time step that is below the threshold level.

\subsection{Euler}

The Euler (multi-material) solver utilizes a fixed mesh, allowing materials to flow (advect) from one element to the next (meshes are fixed in space). Therefore, an external space needs to be modeled. Due to the fixed grid, the Euler method avoids problems of mesh distortion and tangling that are prevalent in Lagrange simulations with large flows. The Euler solver is very well-suited for problems involving extreme material movement, such as fluids and gases. To describe solid behavior, additional calculations are required to transport the solid stress tensor and the history of the material through the grid. Euler is generally more computationally intensive than Lagrange and requires a higher resolution (smaller elements) to accurately capture sharp pressure peaks that often occur with shock waves.

\subsection{ALE}

The ALE method of space discretization is a hybrid of the Lagrange and Euler methods. It allows redefining the grid continuously in arbitrary and predefined ways as the calculation proceeds, which effectively provides a continuous rezoning facility. Various predefined grid motions can be specified, such as free (Lagrange), fixed (Euler), equipotential, equal spacing, and others. The ALE method can model solids as well as liquids.

The advantage of ALE is the ability to reduce and sometimes eliminate difficulties caused by severe mesh distortions encountered by the Lagrange method, thus allowing a calculation to continue efficiently. However, compared to Lagrange, an additional computational step of rezoning is employed to move the grid and remap the solution onto a new grid [6]. 


\section{$3.4 S P H$}

The mesh-free Lagrangian method of space discretization (or SPH method) is a particle-based solver and was initially used in astrophysics. The particles are imbedded in material and they are not only interacting mass points but also interpolation points used to calculate the value of physical variables based on the data from neighboring SPH particles, scaled by a weighting function. Because there is no grid defined, distortion and tangling problems are avoided as well. Compared to the Euler method, material boundaries and interfaces in the SPH are rather well defined and material separation is naturally handled. Therefore, the SPH solver is ideally suited for certain types of problems with extensive material damage and separation, such as cracking. This type of response often occurs with brittle materials and hypervelocity impacts. However, mesh-free methods, such as Smooth Particles Hydrodynamics, can be less efficient than mesh-based Lagrangian methods with comparable resolution.

Fig. 1 gives a short overview of the solver technologies mentioned above. The crucial factor is the grid that causes different outcomes.

The behavior (deflection) of the simple elements is well-known and may be calculated and analyzed using simple equations called shape functions. By applying coupling conditions between the elements at their nodes, the overall stiffness of the structure may be built up and the deflection/distortion of any node and subsequently of the whole structure, can be calculated approximately [15].

Due to the fact that all engineering simulations are based on geometry to represent the design, the target and all its components are simulated as CAD models [16]. Therefore, several runs are necessary: from modeling to calculation to the evaluation and subsequent improvement of the model (Fig. 2).

The most important steps during an FE (finite element) analysis are the evaluation and interpretation of the outcomes followed by suitable modifications of

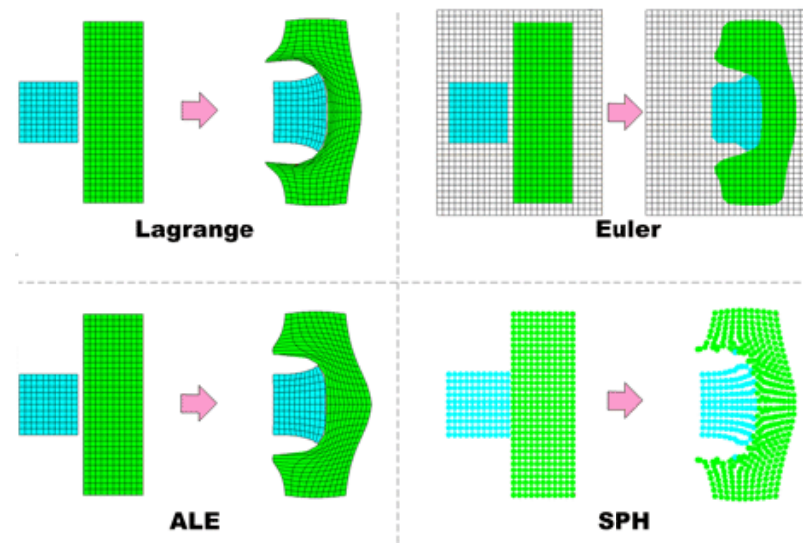

Fig. 1 Examples of Lagrange, Euler, ALE, and SPH simulations on an impact problem [14].

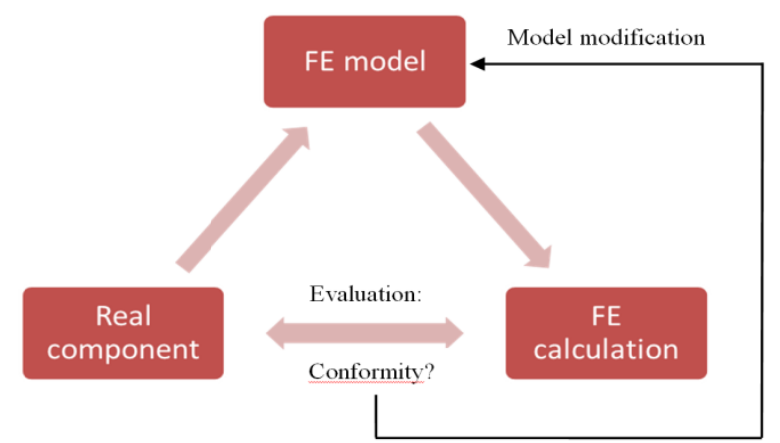

Fig. 2 Iterative procedure of a typical FE analysis [15].

the model. For that reason, ballistic trials are necessary to validate the simulation results. They can be used as the basis of an iterative optimization process.

\section{Ballistic Trials}

Ballistics is an essential component for the evaluation of our results. Here, terminal ballistics is the most important sub-field. It describes the interaction of a projectile with its target. Terminal ballistics is relevant for both small and large caliber projectiles. The task is to analyze and evaluate the impact and its various modes of action. This will provide information on the effect of the projectile and the extinction risk.

In order to develop a numerical model, a ballistic test program is necessary. The ballistic trials are thoroughly documented and analyzed - even fragments must be collected. They provide information about the used armor and the projectile behavior after fire which must be consistent with the simulation results. 
In order to create a data set for the numerical simulations, several experiments have to be performed. Ballistic tests are recorded with high-speed videos and analyzed afterwards. The experimental set-up is shown in Fig. 3. Testing was undertaken at an indoor ballistic testing facility. The target stand provides support behind the target on all four sides. Every ballistic test program includes several trials with different glass laminates. The set-up has to remain unchanged.

The camera system is a pco.dimax that enables fast image rates of 1,279 fps (frames per second) at full resolution of $2,016 \times 2,016$ pixels. The use of a polarizer and a neutral density filter is advisable, so that waves of some polarizations can be blocked while the light of a specific polarization can be passed.

Several targets of different laminate configurations were tested to assess the ballistic limit and the crack propagation for each design. The ballistic limit is considered the velocity required for a particular projectile to reliably (at least $50 \%$ of the time) penetrate a particular piece of material [17]. After the impact, the projectile is examined regarding any kind of change it might have undergone.

Fig. 4 shows a $23 \mathrm{~mm}$ soda lime glass target after testing. The penetrator used in this test was a .44 Remington Magnum, a large-bore cartridge with a lead base and copper jacket. The glass layers showed heavy cracking as a result of the impact.

Close to the impact point is the region of comminution. The comminuted glass is even ejected during the impact. Radial cracks have propagated away from the impact point. The polycarbonate backing layer is deformed up to the maximum bulge height when the velocity of the projectile is close to the ballistic limit. A large amount of the comminuted glass is ejected during the impact. Several targets of different laminate configurations were tested to assess the ballistic limit and the crack propagation for each design.

Cracks propagate with a velocity up to $2,500 \mathrm{~m} / \mathrm{s}$, which is similar to the values in the literature. The

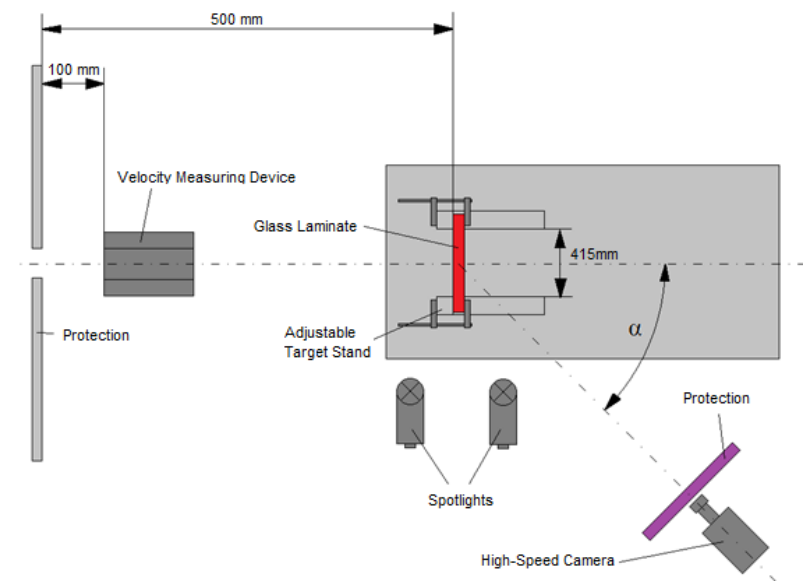

Fig. 3 Experimental set-up.
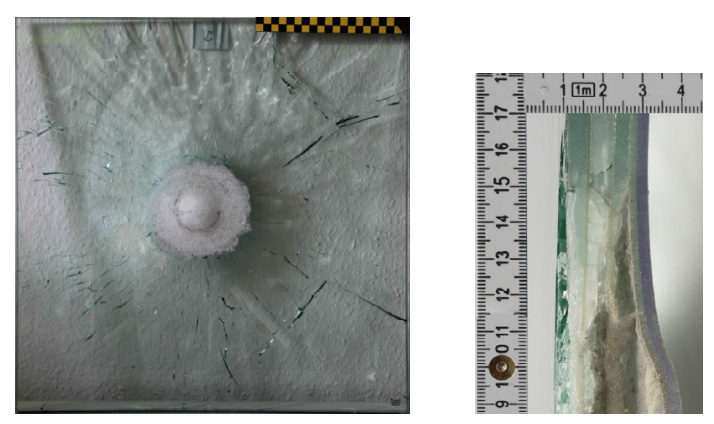

Fig. 4 Trial observation with a $23 \mathrm{~mm}$ glass laminate.

damage of a single glass layer starts with the impact of the projectile corresponding to the depth of the penetration. The polycarbonate layers interrupt the crack propagation and avoid piercing and spalling. The first impact of a .44 Remington cartridge does not cause a total failure of our $23 \mathrm{~mm}$ soda lime glass target. Fragments of the projectile can be found in the impact hole. The last polycarbonate layer remains significantly deformed.

The results of the ballistic tests were provided prior to the simulation work to aid calibration. In this paper, a single trial will illustrate the general approach of the numerical simulations.

\section{Numerical Simulation}

The ballistic tests are followed by computational modeling of the experimental set-up. Then, the experiment is reproduced using numerical simulations. Fig. 5 shows a cross-section of the ballistic glass and the projectile in a CAD model. The geometry and 


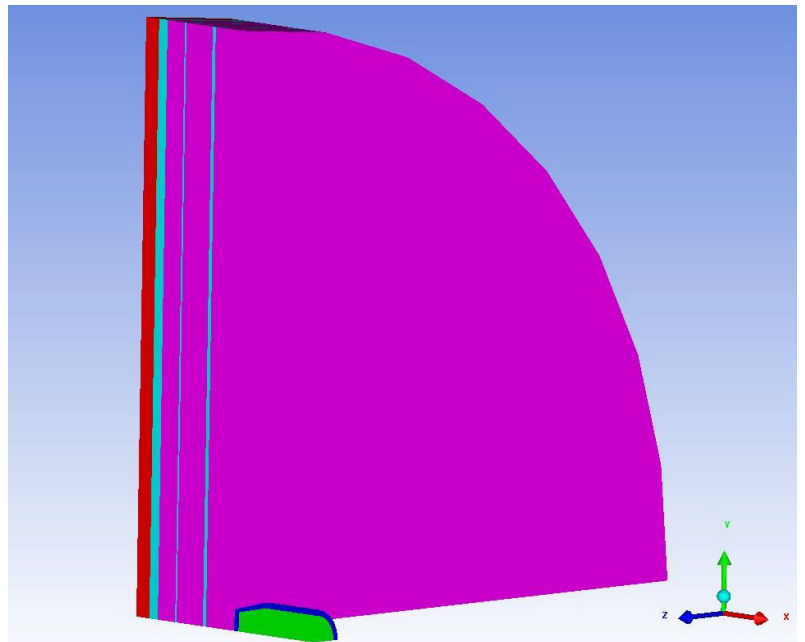

Fig. 5 CAD model.

observed response of the laminate to ballistic impact is approximately symmetric to the axis through the bullet impact point. Therefore, a 2D axisymmetric approach was chosen.

Numerical simulation of transparent armor requires the selection of appropriate material models for the constituent materials and the derivation of suitable material model input data. The laminate systems studied here consist of soda lime float glass, polyurethane interlayer, polyvinyl butyral and polycarbonate. Lead and copper are also required for the .44 Remington Magnum cartridge.

The projectile was divided into two parts - the jacket and the base-which have different properties and even different meshes. These elements have quadratic shape functions and nodes between the element edges. In this way, the computational accuracy as well as the quality of curved model shapes increases. Using the same mesh density, the application of parabolic elements leads to a higher accuracy compared to linear elements (1st order elements).

Different solver technologies have been applied to the soda lime glass laminate. The comparison is presented in the following chapter.

\subsection{Solver Evaluation}

Before the evaluation starts, it has to be noticed that the Euler method is not suitable for numerical simulations dealing with brittle materials. It is generally used for representing fluids and gases, for example, the gas product of high explosives after detonation. To describe solid behavior, additional calculations are required. Cracking cannot be simulated adequately and the computation time is relatively high. For this reason, the Euler (and as a result the ALE) method will not be taken into consideration.

\subsubsection{Lagrange Method}

Fig. 6 shows the simulation with a single Lagrange solver in the first iteration procedure. This method, as mentioned before, is well-suited for representing solids like structures and projectiles. The advantages are computational efficiency and ease of incorporating complex material models. The polyurethane interlayer, polyvinyl butyral and polycarbonate are simulated adequatly. While the soda lime glass also deforms well, the crack propagation cannot be displayed suitably with this solver.

\subsubsection{Mesh Free Lagrangian Method (SPH)}

Before the evaluation starts, it has to be noticed that the Euler method is not suitable for numerical simulations dealing with brittle materials. It is generally used for representing fluids and gases, for example, the gas product of high explosives after detonation. To describe solid behavior, additional calculations are required. Cracking cannot be simulated adequately and the computation time is relatively high. For this reason, the Euler (and as a

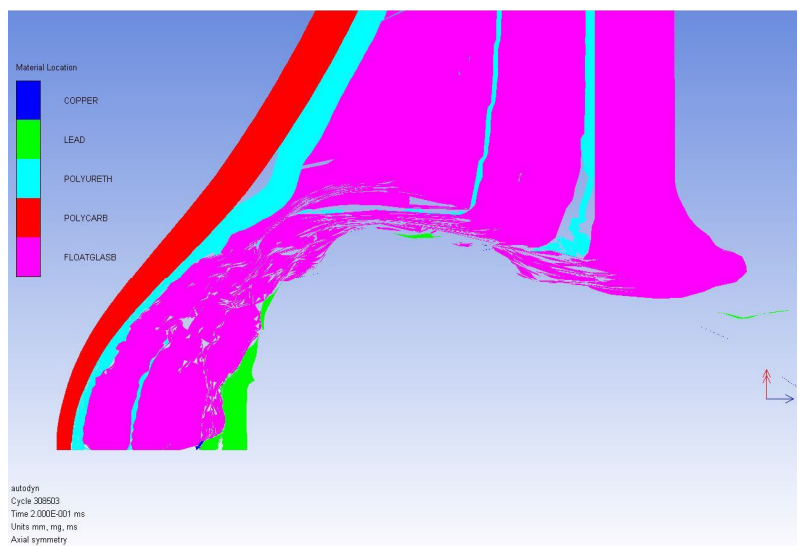

Fig. 6 Lagrange method. 


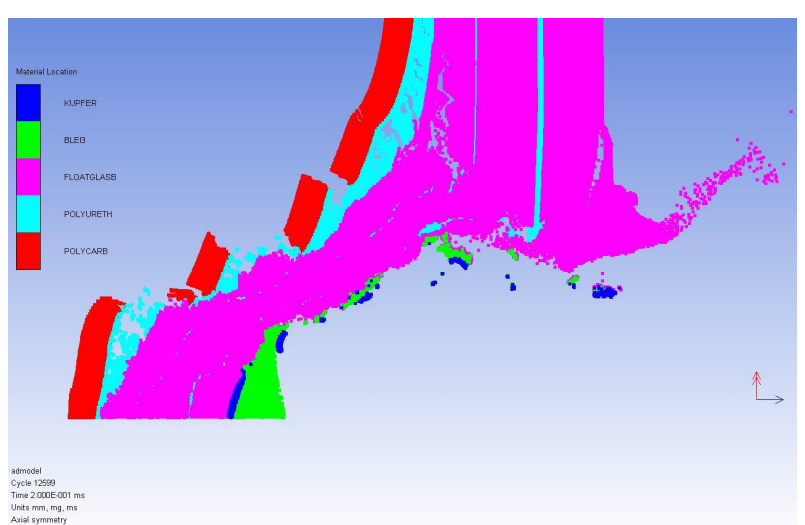

Fig. 7 Mesh free Lagrangian method (SPH).

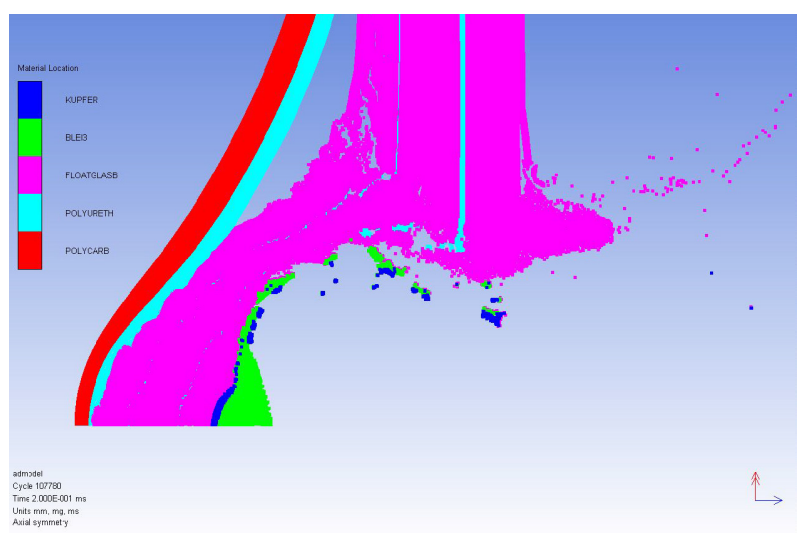

Fig. 8 Coupled multi-solver approach (Lagrange and SPH).

result the ALE) method will not be taken into consideration.

5.1.3 Coupled Multi-solver Approach (Lagrange and $\mathrm{SPH}$ )

Before the evaluation starts, it has to be noticed that the Euler method is not suitable for numerical simulations dealing with brittle materials. It is generally used for representing fluids and gases, for example, the gas product of high explosives after detonation. To describe solid behavior, additional calculations are required. Cracking cannot be simulated adequately and the computation time is relatively high. For this reason, the Euler (and as a result the ALE) method will not be taken into consideration.

\subsection{Simulation Results}

With the coupled multi-solver and optimized material parameters, the simulation results adequately mirror the observations made in the ballistic experiments. Fragmentation and crack propagation are almost equal to the ballistic test shown in Fig. 4.

Fig. 9 illustrates the development of fracture after 10, 20, 50, and $70 \mu \mathrm{s}$ due to shear induced micro-cracking (damage) in the glass during the penetration process.

Note that the failure of the glass in the second and third layers spreads from the glass/polyurethane interlayers back towards the oncoming projectile. This rapid material failure is owed to a reduction in material strength as rarefaction waves from the interface reduce the confining pressure [17].

Small fragments are automatically deleted from the program to reduce computing time. Regarding the protection level of our structures, these fragments are hardly important.

The projectile is subject to a significant deformation. It gets stuck in the target and loses kinetic energy. Fig. 10 compares the numerical simulation of a .44 Remington impact with the experimental result.

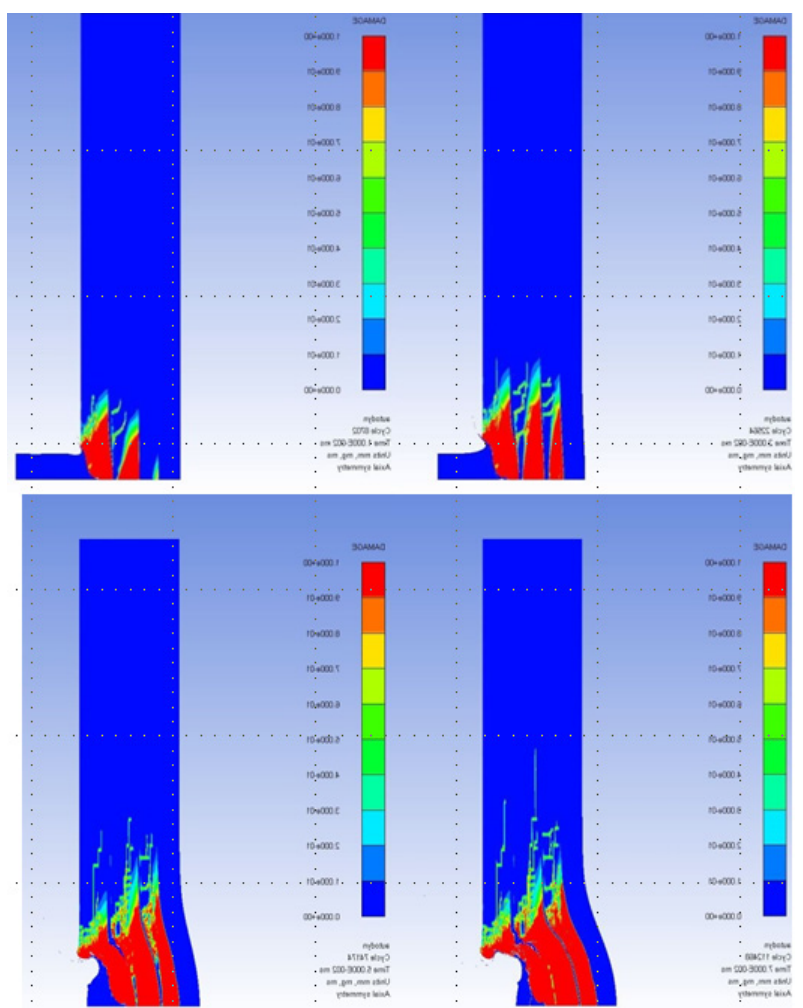

Fig. 9 Crack propagation in a coupled multi-solver simulation model. 

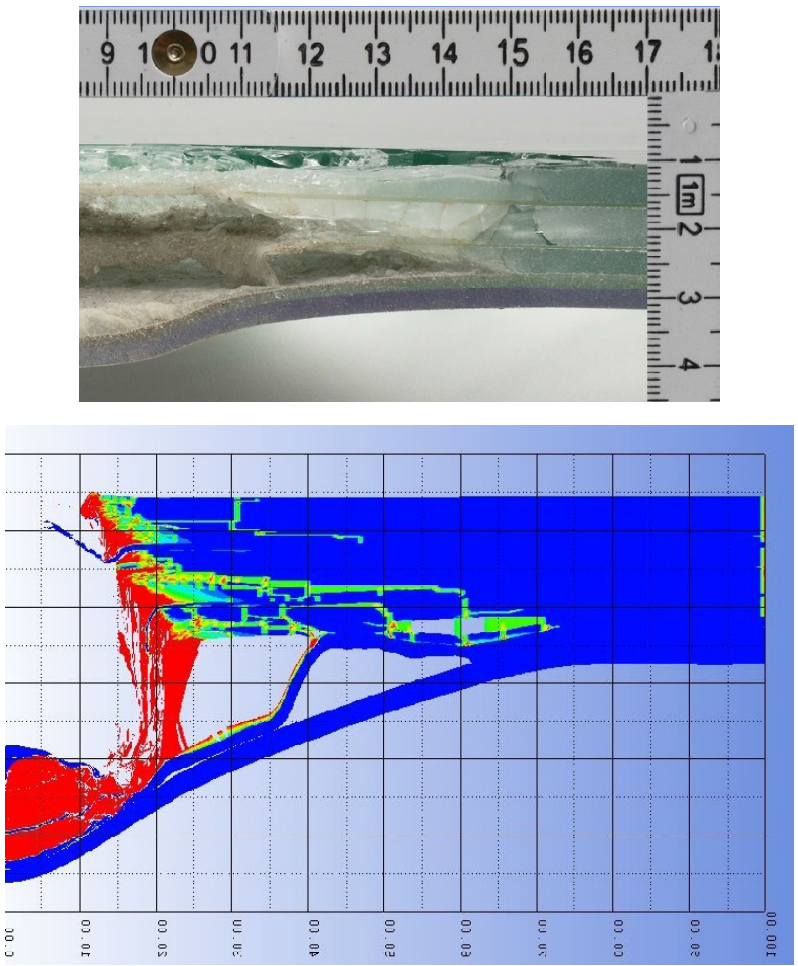

Fig. 10 Comparison between simulation results and ballistic trial.

A clear hole, 45-50 $\mathrm{mm}$ in diameter, is generated in the glass/polyurethane layers of the laminate. A comminuted region of glass, shows highly cracked and completely crushed material, of around $20 \mathrm{~mm}$ in diameter in the first layer which extends to around 120 $\mathrm{mm}$ in diameter in the last layer. Hence, the simulated diameter of comminution is almost identical to that observed experimentally. Even the delamination of the layers can be reproduced in the simulation. The predicted height of the bulge from the flat region of the polycarbonate is $28 \mathrm{~mm}$ compared to approximately 8 $\mathrm{mm}$ observed in the ballistic trials. In the simulation, comminuted glass is caught between the bullet and the polycarbonate layer. This leads to a larger deformation. In reality, comminuted glass is ejected during the impact. The polycarbonate dishes from the edge of the support clamp to form a prominent bulge in the central region. Therefore, reducing the instantaneous geometric erosion strain of the soda lime glass will significantly improve results. Owed to the adopted calibration process, these simulation results correlate well with the experimental observations.

\section{High-Performance Computing}

The objective is to develop and improve the modern armor used in the security sector. To develop better, smarter constructions requires analyzing a wider range of parameters. However, there is a simple rule of thumb: the more design iterations that can be simulated, the more optimized is the final product. As a result, a high-performance computing solution has to dramatically reduce overall engineering simulation time. Beyond the use of HPC, the software is a key strategic enabler of large-scale simulations.

The workload for the above mentioned simulations can be specified as follows:

This research will evaluate the performance of the following server generations:

- HP ProLiant SL390s G7

- HP ProLiant DL580 G7

- HP ProLiant DL380p G8

To take into account the influence of the software, different versions of ANSYS will be applied here. Regarding the Lagrange solver and optimized material parameters in a simplified 2D simulation model (for the purpose of comparison), the following benchmark is obtained for the different simulations:

The results indicate the importance of high-performance computing in combination with competitive simulation software to solve current problems of the computer-aided engineering sector.

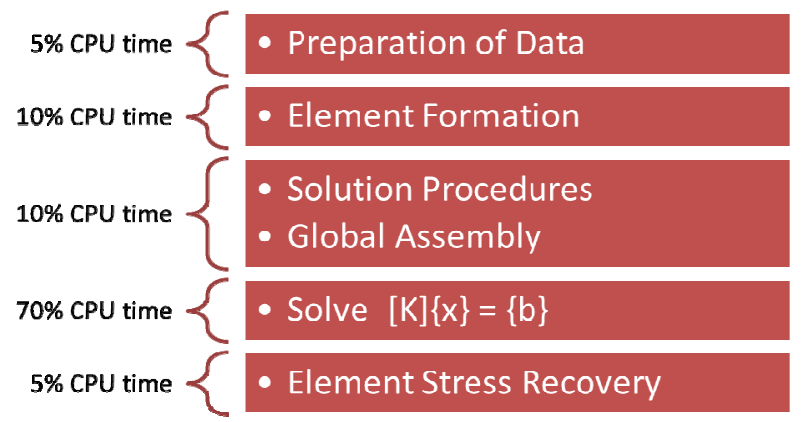

Table 1 Benchmark to illustrate the influence of different server and software generations.

\begin{tabular}{lllllll}
\hline & \multicolumn{2}{c}{ SL390s G7 } & \multicolumn{2}{c}{ DL580 G7 } & \multicolumn{2}{c}{ DL380p G8 } \\
\hline Ver. & 14.5 & 15.0 & 14.5 & 15.0 & 14.5 & 15.0 \\
Time & $35 \mathrm{~m} 02 \mathrm{~s}$ & $18 \mathrm{~m} 59 \mathrm{~s}$ & $27 \mathrm{~m} 08 \mathrm{~s}$ & $16 \mathrm{~m} 19 \mathrm{~s}$ & $21 \mathrm{~m} 47 \mathrm{~s}$ & $12 \mathrm{~m} 55 \mathrm{~s}$ \\
\hline
\end{tabular}




\section{Conclusions}

This work demonstrated how a small number of well-defined experiments can be used to develop, calibrate and validate solver technologies used for simulating the impact of projectiles on complex armor systems and brittle materials.

Existing material models were optimized to reproduce ballistic tests. High-speed videos were used to analyze the characteristics of the projectile before and after the impact. The simulation results demonstrate the successful use of the coupled multi-solver approach. The high level of correlation between the numerical results and the available experimental or observed data demonstrates that the coupled multi-solver approach is an accurate and effective analysis technique.

New concepts and models can be developed and easily tested with the help of modern hydrocodes. The initial design approach of the units and systems has to be as safe and optimal as possible. Therefore, most design concepts are analyzed on the computer. FEM-based simulations are well-suited for this purpose. Here, a numerical model has been developed which is capable of predicting the ballistic performance of soda lime glass/polycarbonate transparent armor systems. Thus, estimates based on experience are being more and more replaced by software.

The gained experience is of prime importance for the development of modern armor. By applying the numerical model a large number of potential armor schemes can be evaluated and the understanding of the interaction between laminate components under ballistic impact can be improved.

\section{References}

[1] Zukas, J. 2014. Introduction to Hydrocodes. Amsterdam: Elsevier Science.

[2] Hamouda, A. M. S., and Hashmi, M. S. J. 1996. "Modelling the Impact and Penetration Events of Modern Engineering Materials: Characteristics of Computer Codes and Material Models." Journal of Materials
Processing Technology 56: 847-62.

[3] Benson, D. J. 1992. "Computational Methods in Lagrangian and Eulerian Hydrocodes." Computer Methods in Applied Mechanics and Engineering 99: 235-394. doi:10.1016/0045-7825(92)90042-I.

[4] Oevermann, M., Gerber, S., and Behrendt, F. 2009. "Euler-Lagrange/DEM Simulation of Wood Gasification in a Bubbling Fluidized Bed Reactor." Particuology 7: 307-16. doi:10.1016/j.partic.2009. 04.004 .

[5] Hicks, D. L., and Liebrock, L. M. 1999. "SPH Hydrocodes Can Be Stabilized with Shape-Shifting." Computers \& Mathematics with Applications 38: 1-16. doi:10.1016/S0898-1221(99)00210-2.

[6] Quan, X., Birnbaum, N. K., Cowler, M. S., and Gerber, B. I. 2003. "Numerical Simulations of Structural Deformation under Shock and Impact Loads Using a Coupled Multi-solver Approach." Presented at the 5th Asia-Pacific Conference on Shock and Impact Loads on Structures, Hunan, China.

[7] Bermeo, N. V., Mendoza, M. G., and Castro, A. G. 2001. "Semantic Representation of CAD Models Based on the IGES Standard." Computer Science 8265: 157-68. doi:10.1007/ 978-3-642-45114-0_13.

[8] Wiederhorn, S. M. 1967. "Influence of Water Vapor on Crack Propagation in Soda-Lime Glass." Journal of the American Ceramic Society 50: 407-14. doi:10.1111/j.1151-2916.1967.tb15145.x.

[9] Richards, M., Clegg, R., and Howlett, S. 1999. "Ballistic Performance Assessment of Glass Laminates through Experimental and Numerical Investigation.” Presented at the 18th International Symposium and Exhibition on Ballistics, San Antonio, Texas, USA.

[10] Pyttel, T., Liebertz, H., and Cai, J. 2011. "Failure Criterion for Laminated Glass under Impact Loading and Its Application in Finite Element Simulation." International Journal of Impact Engineering 38: 252-63. doi:10.1007/s00466-007-0170-1.

[11] Zang, M. Y., Lei, Z., and Wang, S. F. 2007. "Investigation of Impact Fracture Behavior of Automobile Laminated Glass by 3D Discrete Element Method." Computational Mechanics 41: 78-83. doi:10.1007/s00466-007-0170-1.

[12] Collins, G. S. 2002. "An Introduction to Hydrocode Modeling." Applied Modelling and Computation Group, Imperial College London, unpublished.

[13] Stellingwerf, R. F., and Wingate, C. A. 1993. "Impact Modeling with Smooth Particle Hydrodynamics." International Journal of Impact Engineering 14: 707-18.

[14] ANSYS Inc. Available Solution Methods. 
http://www.ansys.com/Products/Simulation+Technology/ Structural+Analysis/Explicit+Dynamics/Features/Availab le+Solution+Methods.

[15] Fröhlich, P. 2005. FEM Application Basics. Berlin: Vieweg Verlag.
[16] Woyand, H. B. 2007. FEM with CATIA V5. Wilburgstetten: J. Schlembach Fachverlag.

[17] Carlucci, D. E., and Jacobson, S. S. 2008. Ballistics: Theory and Design of Guns and Ammunition. New York: CRC Press. 